\title{
Reference Governor for Network Control Systems subject to Variable Time-delay
}

\author{
Stefano Di Cairano $^{\text {a }}$, Uroš V. Kalabić ${ }^{\mathrm{b}}$, Ilya V. Kolmanovsky ${ }^{\mathrm{b}}$ \\ ${ }^{a}$ Mitsubishi Electric Research Laboratories, Cambridge, $M A$ \\ ${ }^{\mathrm{b}}$ Dept. of Aerospace Engineering, Univ. Michigan, Ann Arbor, MI
}

\begin{abstract}
The handling of constraints in systems subject to variable time-delay is a challenging problem. It is particularly relevant to Network Control Systems (NCSs) in which a control system is remotely located with respect to the plant to be controlled. In this paper, we develop reference governors for controlling constrained systems subject to variable delays with a particular focus on the application to NCSs. In the proposed approach, which neither exploits nor depends on any explicit synchronization between the plant and the governor, the closed-loop dynamics are modeled by a sampled data system, for which input delays result in additive disturbances with magnitude proportional to the input rate of change. We first develop a network reference governor (netRG) that guarantees constraint enforcement and finite-time convergence for variable time-delay shorter than the sampling period. Then, we extend the network reference governor to the case of output feedback. Finally, we consider the case of long and potentially unbounded delay. The netRGs is evaluated in a case study of orientation control of a spacecraft with a flexible appendage.
\end{abstract}

Key words: Network Control Systems, Constrained Control, Reference Governors, Time-delay, Remote Control

\section{Introduction}

In applications as diverse as automotive, aerospace, and factory automation, it is appealing to consider the network implementation of control systems in which computing nodes, sensors and actuators, exchange data through a communication network [3-8]. Some of the advantages of these Network Control Systems (NCSs) are higher computational capabilities, reduced cost, increased flexibility and simplified maintenance [6,9-11].

On the other hand, the network medium introduces effects that may cause closed-loop performance degradation $[10,12,13]$. Such effects, that include data losses, variable delays and data corruption, are not commonly dealt with by classical control methods. As a consequence, special design techniques have been developed in the past fifteen years for NCS. In particular, the stability and performance properties of NCSs affected by

\footnotetext{
* This paper was not presented at any IFAC meeting. Preliminary results have been reported in $[1,2]$.

Corresponding author S. Di Cairano.

Email addresses: dicairano@ieee.org (Stefano Di Cairano), kalabic@umich.edu (Uroš V. Kalabić), ilya@umich.edu (Ilya V. Kolmanovsky).
}

data losses, quantization and time delay have been studied extensively, see, e.g., [14-20], and references therein. Also, control methods for dealing with communication network constraints, such as bandwidth and access, have been developed, see, e.g., [21-23].

Another important objective in high performance control systems is the enforcement of constraints on input and output plant variables. In NCS, the network communication introduces a delay that varies and is not known in advance, as it depends, for instance, on the network protocol executions, on the amount of network users, and on environmental conditions. For instance, in [24] a control method for compensating the transmission delay was developed, based on generalized predictive control. In this paper we consider control architectures for constrained control that ensure constraint satisfaction despite the presence of variable time delays. Specifically, we consider a control architecture where a reference governor is remotely located with respect to a plant augmented by a nominal unconstrained controller. A reference governor [25] is a nonlinear state-feedback controller for a constrained plant in closed-loop with a controller that ignores system constraints. The governor receives a desired reference for the closed-loop plant, usually from an operator or a supervisory algorithm, and 
sends to the closed loop plant the closest virtual reference that satisfies the constraints. Ideally, the virtual reference will converge to the desired reference if the latter satisfies the constraints in steady state.

The NCS governor architecture, hereafter referred to as network reference governor (netRG), is suitable when the virtual reference cannot be computed at the plant site, e.g., due to the impossibility or high costs of placing at the plant site a computational unit capable of solving an optimization problem. Some motivating examples for the netRG are in chemical or process control [6], when the plant operates in an extreme environment. Another class of applications is in autonomous spacecraft or remotely piloted UAVs and robots, see, e.g., [5,7,26], where onboard computing hardware capabilities are limited, but there is a communication link with a base station that can host more capable computing hardware. Other applications include automotive vehicles [27], where networks connect microcontrollers and actuators with varying levels of local computing capabilities.

NCS architectures based on reference governors have been previously proposed for enforcing constraints in presence of delays and data losses. In the early paper [28], a prediction scheme with additional logic was used to account for a delay bounded over a given horizon, and a recovery algorithm was developed for cases when the delay was longer than expected. In [29], this approach was extended by algorithms based on data redundancy that allow for multiple interconnected systems to be controlled through the network with increased robustness to delays and data losses. In both approaches, the delay is assumed to be a multiple of the sampling period, as is the case in synchronous architectures, where the plant and controller are synchronized by a common clock. Thus, a purely discrete-time model of the plant is exploited for predictive constraint enforcement.

In this paper we consider netRG strategies for systems affected by a variable time delay that may take continuous values in a (possibly unbounded) interval. This kind of delay occurs in NCS with asynchronous communication protocols, such as the CAN protocol used in automotive. We focus on the case where the delay affects the command sent from the netRG to the plant, and we derive a sampled-data system model, where the effect of the delay is modeled as an additive disturbance with magnitude proportional to the rate of change of the command. By using robust constraints, we first provide a netRG that guarantees constraint enforcement for a delay that is always smaller than the sampling period, and we show that the finite-time convergence properties of the standard governor $[30,31]$ are preserved. The netRG is then extended to the case of imperfect state information, where an observer is used for state estimation, and a bound on the estimation error is used to robustly enforce constraints. Finally, we present a netRG that can deal with a longer and possibly unbounded delay, where a simple acceptance/rejection logic is implemented at the plant.

The netRG modulates the speed of convergence towards the desired reference so that the uncertain delay does not cause constraint violations. Thus, it differs from $[28,29]$, and instead exploits an idea similar to [32], while the type and sources of uncertainty and methods for addressing it are significantly different. Some of the advantages of the proposed netRG are that it does not require synchronization between the plant and the governor, that it has computational burden similar to standard governors, that it allows for output feedback, and that it is robust to intersampling actuation, which may occur due to network and computational jitter. While mainly driven by applications in NCSs, the netRG concepts are applicable to many systems subject to time-varying delays, such as those in automotive and aerospace applications.

The paper is structured as follows. In Section 2 we introduce the NCS model, the reference governor strategy and the model of the network-induced time delay. In Section 3 we formulate the netRG for the case where the time-varying network delay is "short", which here means, shorter than the sampling period. In Section 4 we consider the case of output feedback, where an estimator is used to reconstruct the state. Finally, in Section 5 the control strategy is extended to handle "long" delays, that is, longer than the sampling period. The netRG is applied under different conditions to the case study of attitude control of a spacecraft with a flexible appendage in Section 6. The conclusions are summarized in Section 7.

Notation. We denote the real, nonnegative real, positive real, and integer, nonnegative integer, positive integers by $\mathbb{R}, \mathbb{R}_{0+}, \mathbb{R}_{+}$, and $\mathbb{Z}, \mathbb{Z}_{0+}, \mathbb{Z}_{+}$, respectively. Furthermore, $\mathbb{Z}_{[a, b]} \triangleq\{z \in \mathbb{Z}: a \leq z \leq b\}$, and a similar notation is also used with $\mathbb{R}$. Given a continuous signal $a(t)$ sampled with sampling period $T_{s}$, we denote by $a(k)$ the value at the $k^{t h}$ sampling instant, that is $a(k)=a\left(t_{k}\right)$, where $t_{k}=k T_{s}$. Where $a$ is a vector, $\|a\|_{p}$ indicates the $p$-norm of the vector, while when $A$ is a matrix, $\|A\|_{p}$ indicates the induced matrix $p$-norm. When $a$ is a vector, $[a]_{i}$ is the $i^{t h}$ component of the vector. We denote the interior of the set $\mathcal{X}$ by $\operatorname{int}(\mathcal{X})$, its convex hull by $\operatorname{co}(\mathcal{X})$, and the Minkowski sum of sets $\mathcal{X}$ and $\mathcal{Y}$ by $\mathcal{X} \oplus \mathcal{Y}$. The $\infty$-norm ball of radius $\rho$ in $\mathbb{R}^{n}$ is denoted by $\mathcal{B}(\rho)$, and the vertices of $\mathcal{B}(1)$, i.e., $\mathcal{X}=\left\{x \in \mathbb{R}^{n}:\|x\|_{\infty} \leq 1\right\}$, are denoted by $\left\{\eta_{i}\right\}_{i=1}^{n_{\eta}}$, where $n_{\eta}=2^{n} . \mathbf{P}[\xi]$, where $\xi$ is a generic event, indicates the probability of $\xi$.

\section{NCS Model and Problem Definition}

In this section we introduce the NCS and the impact of the network-induced delay on the closed-loop system. 


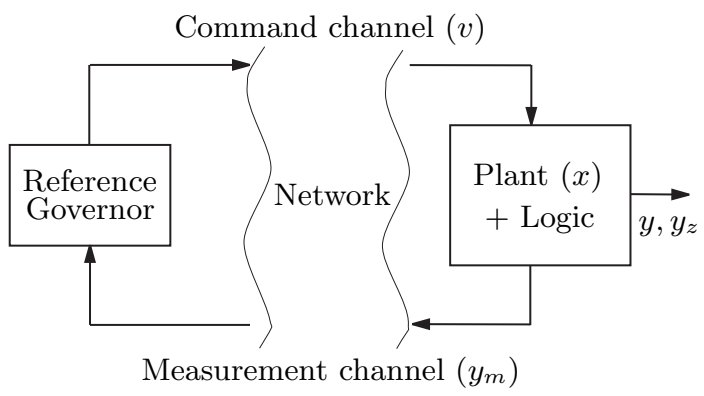

Fig. 1. Schematics of a NCS based on reference governor.

\subsection{Network control system model}

We consider the NCS shown in Figure 1, where a reference governor is connected to a plant, possibly augmented by a local stabilizing controller, through a communication network. The reference governor sends commands to the plant, through the command (forward) channel and receives measurements from the plant through the measurement (feedback) channel. The command and measurement channels are logical channels, that may share the same physical network infrastructure. The signals sent through the network are sampled, hence the transmitter also operates as a sampler, and the receiver operates also as a zero order hold.

The communication network causes a time-varying delay between the sent and received signals. We assume no time-stamping nor sequential indexing of the data packets that results in more involved communication protocols. Consider a sequence $\left\{a_{k}\right\}_{k}, k \in \mathbb{Z}_{0+}$, where $a_{k}$ is sent at time $t_{k} \in \mathbb{R}_{0+}$ and received at time $t_{k}+\delta_{k}$, $\delta_{k} \in \mathbb{R}_{0+}$. The receiver output is the signal

$$
a(t)=a_{k(t)}, \quad k(t)=\arg \max _{\substack{k \in \mathbb{Z}_{0+}: \\ t_{k}+\delta_{k} \leq t}} t_{k}+\delta_{k} .
$$

In (1) the receiver output at time $t$ is the last received signal, which may not be the latest sent packet. In the simpler case where $\delta_{k} \in \mathbb{R}_{\left[0, t_{k+1}-t_{k}\right)}$, the receiver output

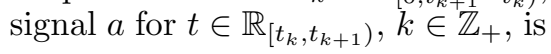

$$
a(t)= \begin{cases}a_{k-1} & \text { if } t \in \mathbb{R}_{\left[t_{k}, t_{k}+\delta_{k}\right)}, \\ a_{k} & \text { if } t \in \mathbb{R}_{\left[t_{k}+\delta_{k}, t_{k+1}\right)}\end{cases}
$$

In this paper, we first develop control strategies for the case where the delay is modelled by (2), then we extend our approach to the more general case (1). Also, we focus on the case where the delay induced by the network affects only the command channel.

The plant to be controlled is the continuous-time linear time-invariant dynamical system,

$\dot{x}(t)=A_{c} x(t)+B_{c} r(t)$,

$y(t)=C_{c} x(t)$,

where $x \in \mathbb{R}^{n}$ is the state vector, $y \in \mathbb{R}^{p}$ is the constraint output vector, and $r \in \mathbb{R}^{m}$ is the input vector, which, in this context, is the reference selected to achieve the desired equilibrium. In this paper, $y$ is used to formulate the constraints specified as

$$
y \in \mathcal{C} \subset \mathbb{R}^{p},
$$

where $\mathcal{C}$ is a given compact polyhedron, and $0 \in \operatorname{int}(\mathcal{C})$.

Remark 1 Model (3) represents a plant possibly in closed-loop with a co-located controller. In NCSs, the co-located controller may be a computationally simple controller (e.g., a PID) aimed at asymptotically stabilizing the physical plant and achieving a unitary steady state gain. If the co-located controller is discrete-time, it is assumed that its sampling period is such that (3) is a valid approximation of the closed-loop behavior from the reference governor perspective.

In this paper we develop netRGs that enforce (4) pointwise in time. In the design, we exploit a sampled data model of (3) with period $T_{s} \in \mathbb{R}_{+}$being the update period of the netRG,

$$
\begin{aligned}
x(k+1) & =A x(k)+B v(k), \\
y(k) & =C x(k),
\end{aligned}
$$

where $A=e^{A_{c} T_{s}}, B=\int_{0}^{T_{s}} e^{A_{c}\left(T_{s}-\tau\right)} B_{c} d \tau, C=C_{c}$, and the reference $r$ in (3) is substituted by a virtual reference $v \in \mathbb{R}^{m}$ generated by the netRG so that the pointwise in time constraints,

$$
y(k) \in \mathcal{C}, \forall k \in \mathbb{Z}_{+},
$$

are enforced and $v$ is "as close as possible" to $r$.

Assumption 2 Systems (3) and (5) are asymptotically stable, i.e., $A_{c}$ is Hurwitz, and $A$ is Schur. The pair $(A, C)$ is observable.

Finally, the measurement vector $y_{m} \in \mathbb{R}^{p_{m}}$, which is fed back from the plant is,

$$
y_{m}(k)=C_{m} x(k) .
$$

Assumption $3 C_{m}$ in (7) is such that $\operatorname{rank}\left(C_{m}\right)=n$, i.e., given $y_{m}(k), x(k)$ is uniquely determined.

By Assumption 3, the full state $x(k)$ is known. We discuss the case when Assumption 3 does not hold later, in Section 4. 


\subsection{Reference governor}

Next, we recall basic concepts and results of the conventional reference governor, when $\delta_{k}=0$ for all $k \in \mathbb{Z}_{0+}$.

Definition 4 Given $x(k+1)=f(x(k)), x \in \mathbb{R}^{n}, \mathcal{X} \subseteq$ $\mathbb{R}^{n}$ is positive invariant $(P I)$ if for all $x \in \mathcal{X}, f(x) \in \mathcal{X}$.

Definition 5 ( [33]) The maximum output admissible set $\mathcal{O}_{\infty} \subset \mathbb{R}^{n+m}$ for (5), (6) for constant reference $v(k+$ $1)=v(k)$ is the PI set,

$$
\begin{aligned}
& \mathcal{O}_{\infty} \triangleq\{(x, v) \in \mathbb{R}^{n+m}: x(k)=x, v(h)=v, \\
&\left.\forall h \in \mathbb{Z}_{[k, \infty)} \Longrightarrow y(h) \in \mathcal{C}, \forall h \in \mathbb{Z}_{[k, \infty)}\right\} .
\end{aligned}
$$

Definition 6 Denote the steady state of the constant command $v \in \mathbb{R}^{m}$ by $x_{e}(v) \in \mathbb{R}^{n}$. The set $\Gamma \subseteq\left\{v \in \mathbb{R}^{m}: C x_{e}(v) \in \operatorname{int}(\mathcal{C})\right\}$ is a compact polyhedral set of strictly steady state admissible references, i.e., $\left(x_{e}(v), v\right) \in \operatorname{int}\left(\mathcal{O}_{\infty}\right)$ for all $v \in \Gamma$.

Lemma 7 ( $[\mathbf{3 0}, \mathbf{3 3}])$ Let Assumption 2 hold. $\mathcal{O}_{\infty}$ is convex, compact, and it has an arbitrarily close PI inner approximation $\tilde{\mathcal{O}}_{\infty} \subseteq \mathcal{O}_{\infty}$ described by a finite number of linear inequalities, i.e., there exists a finite $q \in \mathbb{Z}_{+}$, $H_{x} \in \mathbb{R}^{q \times n}, H_{v} \in \mathbb{R}^{q \times p}, K \in \mathbb{R}^{q}$ such that,

$$
\tilde{\mathcal{O}}_{\infty}=\left\{(x, v): H_{x} x+H_{v} v \leq K\right\}
$$

In what follows, we disregard the arbitrarily small difference between $\tilde{\mathcal{O}}_{\infty}$ and $\mathcal{O}_{\infty}$, which affect only the computations, and with a little abuse we consider $\tilde{\mathcal{O}}_{\infty}=\mathcal{O}_{\infty}$.

The reference governor $[25,30,33]$ is a control law $v=$ $g(x, r)$ where,

$$
\begin{aligned}
g(x, r)=\arg \min _{v \in \Gamma} & \|r-v\|_{2}^{2} \\
\text { s.t. } & (A x+B v, v) \in \mathcal{O}_{\infty},
\end{aligned}
$$

so that the virtual reference (or command) $v$ is the projection of $r$ onto the section of $\mathcal{O}_{\infty}$ at the next state.

Theorem 8 ( [30]) Consider (5) with $v(k)=g(x(k), r(k))$ defined by $(10)$, and let $r(k)=r$ for all $k \in \mathbb{Z}_{0+}$. Then: (i) the set $\mathcal{X}_{\text {feas }}=\left\{x \in \mathbb{R}^{n}: \exists v,(A x+B v, v) \in \mathcal{O}_{\infty}\right\}$ is PI for (5), (10); (ii) if at some $\bar{h} \in \mathbb{Z}_{0+}, x(\bar{h}) \in \mathcal{X}_{\text {feas }}$, $y(k) \in \mathcal{C}$ for all $k \in \mathbb{Z}_{[\bar{h}, \infty)} ;($ iii $)$ if $r \in \Gamma$, there exists a finite $\bar{k} \in \mathbb{Z}_{+}$such that $v(\bar{k})=r$.

Remark 9 The pointwise-in time constraints can be enforced on a shorter sampling period than $T_{s}$ by extending $y$ in (5) to include intersampling instants. Enforcing constraints with period $T_{s} / n_{c}, n_{c} \in \mathbb{Z}_{+}$, results in $y \in \mathbb{R}^{p \cdot n_{c}}$.

\subsection{Control-oriented model of plant subject to delay}

Let the network in Figure 1 induce a delay $\delta_{k}$ in the command (forward) channel, while the measurement (feedback) channel has no delay.

Lemma 10 Let $\delta_{k} \in \mathbb{R}_{[0, \bar{\delta}]}$ for all $k \in \mathbb{Z}_{0+}, \bar{\delta}<T_{s}$. Then,

$$
x(k+1)=A x(k)+B v(k)+W\left(\delta_{k}\right) \Delta v(k),
$$

where $\Delta v(k)=v(k)-v(k-1)$, and,

$$
W(\delta)=A_{c}^{-1}\left(e^{A_{c}\left(T_{s}-\delta\right)}-e^{A_{c} T_{s}}\right) B_{c} .
$$

Proof 1 Consider $v\left(t_{k}\right)$ applied at $t_{k}+\delta_{k}$, where $\delta_{k} \in$ $\mathbb{R}_{[0, \bar{\delta}]}$. Based on $(2)$ with $a(t)=v(t)$ and $(3)$,

$$
\begin{aligned}
x\left(t_{k+1}\right)= & e^{A_{c} T_{s}} x\left(t_{k}\right)+\int_{0}^{\delta_{k}} e^{A_{c}\left(T_{s}-\tau\right)} B_{c} v\left(t_{k-1}\right) d \tau \\
& +\int_{\delta_{k}}^{T_{s}} e^{A_{c}\left(T_{s}-\tau\right)} B_{c} v\left(t_{k}\right) d \tau .
\end{aligned}
$$

By the definition of $A, B, x\left(t_{k+1}\right)=A x\left(t_{k}\right)+B v\left(t_{k}\right)+$ $\int_{0}^{\delta_{k}} e^{A_{c}\left(T_{s}-\tau\right)} B_{c} v\left(t_{k-1}\right)-\int_{0}^{\delta_{k}} e^{A_{c}\left(T_{s}-\tau\right)} B_{c} v\left(t_{k}\right) d \tau$, and we obtain (11) with $W(\delta)=-\int_{0}^{\delta} e^{A_{c}\left(T_{s}-\tau\right)} B_{c} d \tau$. Thus, (12) follows by integration, since $A_{c}$ is Hurwitz, and hence invertible.

By (11), a delay in the command channel modifies the plant dynamics. Figure 2 shows the response of a second order system controlled by a reference governor that enforces constraints but does not account for the delay. The command is subject to a random delay $\delta_{k} \in\left[0,0.99 T_{s}\right]$, and we set $v(t)=v(t-1)$ if no feasible solution to (10) exists at time $t$. Figure 2 shows that the constraints are violated because of the time delay.

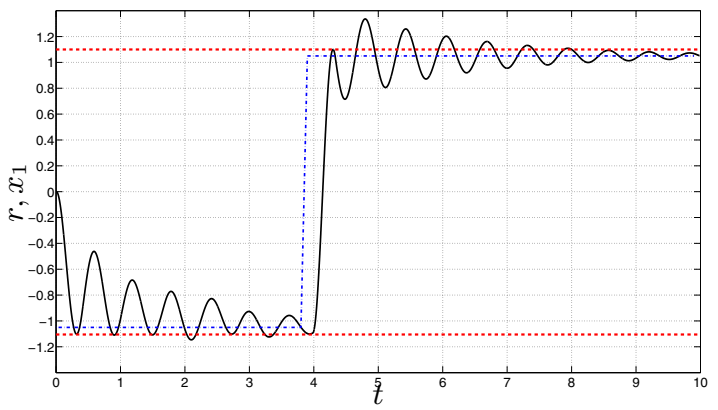

Fig. 2. Constraint violations on a system controlled by a reference governor due to variable delay. Position (solid), position reference (dash-dot), and constraints (dash).

Equation (13) is similar to that in [18], although there the authors continue towards computing a difference inclusion, while here we obtain an equation for characterizing the disturbance. 
For the relatively benign case when $\delta_{k}$ is known at $t_{k} \in$ $\mathbb{R}_{0+}$, for every $k \in \mathbb{Z}_{0+}$, the reference governor can be modified to account for it.

Theorem 11 Consider (5a) where for all $k \in \mathbb{Z}_{+}$the command signal $v(k)$ is subject to delay (2) with the current value of the delay $\delta_{k}<T_{s}$ known for all $k \in \mathbb{Z}_{0+}$. Consider the reference governor law,

$$
v(k)=g_{\delta}\left(x(k), r(k), v(k-1), \delta_{k}\right),
$$

where $g_{\delta}: \mathbb{R}^{n} \times \mathbb{R}^{m} \times \mathbb{R}^{m} \times \mathbb{R}_{0+} \rightarrow \mathbb{R}^{m}$ is defined by,

$$
\begin{aligned}
& g_{\delta}\left(x, r, v^{-}, \delta\right)= \\
& \arg \min _{v \in \Gamma}\|r-v\|_{2}^{2} \\
& \text { s.t. }(A x+B v+W(\delta) \Delta v, v) \in \mathcal{O}_{\infty}
\end{aligned}
$$

for $\Delta v=v-v^{-}$, and $W(\delta)$ as in Lemma 10.

If at some $\bar{h} \in \mathbb{Z}_{0+},(x(\bar{h}), v(\bar{h}-1)) \in \mathcal{O}_{\infty}$, then for $(11)$ in closed loop with $(14), y(k) \in \mathcal{C}$ for all $t \in \mathbb{Z}_{[k, \infty)}$.

Proof 2 If $(x(\bar{h}), v(\bar{h}-1)) \in \mathcal{O}_{\infty}$, and since by (11) the delay does not have an effect when $\Delta v(k)=0, v(\bar{h})=$ $v(\bar{h}-1)$ is feasible for (15). Since $\delta_{\bar{h}}$ is known, $v(\bar{h})$ generated from (14) is such that $(x(\bar{h}+1), v(\bar{h})) \in \mathcal{O}_{\infty}$. Since $\mathcal{O}_{\infty}$ is $P I$, the reasoning can be repeated for all $k \in \mathbb{Z}_{[\bar{h}, \infty)}$. Thus, $(x(k), v(k-1)) \in \mathcal{O}_{\infty}$, and hence $y(k) \in \mathcal{C}$, for all $k \in \mathbb{Z}_{[\bar{h}, \infty)}$.

By Definition 5 , the delay does not affect $\mathcal{O}_{\infty}$, but only the computation of $v$ in (15).

An immediate extension of Theorem 11 is for when the delay takes one of a finite number of known possible values, but it is otherwise unknown. In this case (15b) can be replicated for each possible delay value. Computationally, this approach becomes unwieldy if the number of possible delay values is large.

To account for the case where $\left\{\delta_{k}\right\}_{k=0}^{\infty}$ is unknown and can take arbitrary values, as in asynchronous NCSs, the reference governor must be further modified.

\section{Network Reference Governor for Short Delay}

Next, we consider a network delay $\delta_{k}$ that is variable and unknown. Since Theorem 11 does not apply, (14), (15) must be modified to deal with the uncertainty in the delay. First, we consider $\delta_{k}<T_{s}$ for all $k \in \mathbb{Z}_{0+}$, i.e., the delay is shorter than the netRG update period, and (2) can be used. For this case, we introduce the following assumption.

Assumption 12 There exists $\bar{\delta} \in \mathbb{R}_{\left[0, T_{s}\right)}$ such that $\delta_{k} \in$ $\mathbb{R}_{[0, \bar{\delta}]}$ for all $k \in \mathbb{Z}_{0+}$.
Under Assumption 12 phenomena such as command overtaking, i.e., a later command being received earlier than a previously sent command, do not occur. However, since the netRG commands a reference, $T_{s}$ may be long with respect to the system time constant, and hence even under Assumption 12 the delay can cause significant constraint violations, if ignored.

Remark 13 The case when the delay has a constant component, i.e., $\delta_{k} \in \mathbb{R}_{\left[\delta_{c}, \delta_{c}+\bar{\delta}\right]}$, can be dealt with by integrating the system forward for a time $\delta_{c}$, and by considering a variable delay $\delta_{k} \in \mathbb{R}_{[0, \bar{\delta}]}$ acting from the predicted state, see, e.g., [2].

From (11) we formulate the system model subject to unknown command delay as,

$$
\begin{aligned}
& x(k+1)= A x(k)+B v(k)+d(k), \\
& d(k) \in \mathcal{D}(\Delta v(k)), \\
& \mathcal{D}(\Delta v)=\left\{d \in \mathbb{R}^{n}: d=W(\delta) \Delta v, \delta \in \mathbb{R}_{[0, \bar{\delta}]}\right\} .
\end{aligned}
$$

When $\delta_{k}$ is unknown, $d(k)$ in (16) acts as a disturbance bounded in the set $\mathcal{D}(\Delta v(k))$. Hence, we modify (10) to ensure constraint enforcement for any admissible delay,

$$
\begin{aligned}
& g_{d}\left(x, r, v^{-}\right)=\min _{v \in \Gamma}\|r-v\|_{2}^{2} \\
& \text { s.t. }(A x+B v+d, v) \in \mathcal{O}_{\infty}, \\
& \forall d \in \mathcal{D}(\Delta v),
\end{aligned}
$$

where $\Delta v=v-v^{-}$. The control law (17) ensures that for any admissible delay the state will stay in the maximum output admissible set, but it is not computationally tractable due to (17c). The netRG is based on a computationally efficient approximation of (17).

Lemma 14 Under Assumption 12, consider (11) and the $\mathcal{O}_{\infty}$ set for (5), and let,

$$
\bar{W}=\max _{\delta \in \mathbb{R}_{[0, \bar{\delta}]}}\|W(\delta)\|_{\infty}
$$

Let the netRG law $g_{w}: \mathbb{R}^{n} \times \mathbb{R}^{m} \times \mathbb{R}^{m}$ be,

$$
\begin{aligned}
g_{w}\left(x, r, v^{-}\right) & = \\
\arg \min _{v \in \Gamma} & \|r-v\|_{2}^{2} \\
\text { s.t. } & \left(A x+B v+\bar{W} \eta_{i}\|\Delta v\|_{\infty}, v\right) \in \mathcal{O}_{\infty}, \\
& \forall i \in \mathbb{Z}_{\left[1, n_{\eta}\right]} .
\end{aligned}
$$

Given $x(k), r(k), v(k-1)$, with $(x(k), v(k-1)) \in \mathcal{O}_{\infty}$, $v(k)=g_{w}(x(k), r(k), v(k-1))$ is such that for any $\delta_{k} \in$ $\mathbb{R}_{[0, \bar{\delta}]},(x(k+1), v(k)) \in \mathcal{O}_{\infty}$.

Proof 3 (17b), (17c) can be reformulated as,

$$
(A x+B v \oplus \mathcal{D}(\Delta v), v) \subseteq \mathcal{O}_{\infty} .
$$


Since by the norm properties $\bar{W}\|\Delta v\|_{\infty} \geq\|W(\delta) \Delta v\|_{\infty}$ for all $\delta \in \mathbb{R}_{[0, \bar{\delta}]}$, it follows that $\mathcal{D}(\Delta v) \subseteq \tilde{\mathcal{D}}(\Delta v)$ where,

$\tilde{\mathcal{D}}(\Delta v) \triangleq\left\{d \in \mathbb{R}^{n}:\|d\|_{\infty} \leq \bar{W}\|\Delta v\|_{\infty}\right\}$.

According to (21), $\tilde{\mathcal{D}}(\Delta v)$ is a (convex) polytope. By the definition of $\infty$-norm, $\tilde{\mathcal{D}}(\Delta v)=\operatorname{co}\left(\left\{\eta_{i} \bar{W}\|\Delta v\|_{\infty}\right\}_{i=1}^{n}\right)$ and by convexity of $\mathcal{O}_{\infty}$ and $\tilde{\mathcal{D}}(\Delta v)$,

$$
\begin{aligned}
\left(A x+B v+\eta_{i} \bar{W}\|\Delta v\|, v\right) \in \mathcal{O}_{\infty}, \quad \forall i \in \mathbb{Z}_{\left[1, n_{\eta}\right]} \\
\Longrightarrow(A x+B v+d, v) \in \mathcal{O}_{\infty}, \forall d \in \tilde{\mathcal{D}}(\Delta v) .
\end{aligned}
$$

Since $\mathcal{D}(\Delta v) \subset \tilde{\mathcal{D}}(\Delta v),(17 \mathrm{~b}),(17 \mathrm{c})$ are enforced and $(A x+B v+d, v) \in \mathcal{O}_{\infty}$ for all $\delta \in \mathbb{R}_{[0, \bar{\delta}]}$. Finally, a feasible reference $v$ exists since $v=v(k-1)$ is such that $\mathcal{D}(\Delta v)=\{0\}$ and $(A x(k)+B v, v) \in \mathcal{O}_{\infty}$.

The netRG (19) enforces the constraints for the set of possible next states, obtained as a nominal evolution for $\delta=0$, plus a delay-induced uncertainty set. Also, when replacing $\mathcal{D}(\Delta v)$ by $\tilde{\mathcal{D}}(\Delta v)$ in $(17 \mathrm{c})$, the recursive feasibility of $v=v^{-}$in (19) is maintained since $\tilde{\mathcal{D}}(0)=$ $\mathcal{D}(0)$.

Remark 15 While making the optimization problem computationally feasible, (19c) introduces some conservativeness due to the approximation of $\mathcal{D}(\Delta v)$. Tighter approximations can be found, for instance based on general polytopes [18]. For instance, for all $i \in \mathbb{Z}_{[1, m]}$, let $h_{i} \in \mathbb{Z}_{+}$and $\omega_{i}^{(j)}, j \in \mathbb{Z}_{\left[1, h_{i}\right]}$ be such that $[W(\delta)]_{i} \subseteq \operatorname{co}\left(\left\{\omega_{i}^{(j)}\right\}_{j=1}^{h_{i}}\right)$, for all $\delta \in \mathbb{R}_{[0, \bar{\delta}]}$. Define $\tilde{\mathcal{D}}_{i}\left([\Delta v]_{i}\right)=\operatorname{co}\left(\left\{\omega_{i}^{(j)}[\Delta v]_{i}\right\}_{j=1}^{h_{i}}\right)=\operatorname{co}\left(\left\{\omega_{i}^{(j)}\right\}_{j=1}^{h_{i}}\right)[\Delta v]_{i}$, then $\mathcal{D}(\Delta v) \subseteq \bigoplus_{i=1}^{m} \tilde{\mathcal{D}}_{i}\left([\Delta v]_{i}\right)$. Thus, $(17 \mathrm{~b})$, (17c) are guaranteed by enforcing $\left(A x+B v+\sum_{i=1}^{m} \omega_{i}^{\left(j_{i}\right)}[\Delta v]_{i}, v\right) \in$ $\mathcal{O}_{\infty}$ for all $j_{i} \in \mathbb{Z}_{\left[1, h_{i}\right]}, i \in \mathbb{Z}_{[1, m]}$.

The netRG (19) can be evaluated by solving a quadratic program. Under Assumption $2, \mathcal{O}_{\infty}$ is defined by the polytope (9), and substituting (9) into (19) and introducing the auxiliary variable $\xi \in \mathbb{R}_{0+}$,

$$
\begin{aligned}
& \left(g_{w}\left(x, r, v^{-}\right), \xi\right)= \\
& \arg \min _{v \in \Gamma, \xi}\|r-v\|_{2}^{2} \\
& \text { s.t. } H_{x}\left(A x+B v+\eta_{i} \bar{W} \xi\right)+H_{v} v \leq K, \\
& \quad \xi \geq\left[v-v^{-}\right]_{j}, \\
& \quad \xi \geq-\left[v-v^{-}\right]_{j}, \\
& \quad i \in \mathbb{Z}_{\left[1, n_{\eta}\right]}, \quad j \in \mathbb{Z}_{[1, m]},
\end{aligned}
$$

where at optimum $\xi=\Delta v$, and (23c) enforces $(x(k+$ $1), v(k)) \in \mathcal{O}_{\infty}$ for the vertices of $\tilde{\mathcal{D}}(\Delta v)$, thus enforcing (17b) for all $d \in \tilde{\mathcal{D}}(\Delta v) \supset \mathcal{D}(\Delta v)$.
The following theorem summarizes the properties of the network reference governor (19).

Theorem 16 Consider (5) under Assumption 2, and let the command $v(k)$ be subject to random delay satisfying Assumption 12. If at some $\bar{h} \in \mathbb{Z}_{0+},(x(\bar{h}), v(\bar{h}-$ $1)) \in \mathcal{O}_{\infty}$, then for the closed loop of (5) with $v(k)=$ $g_{w}(x(k), r(k), v(k-1))$, it follows that $y(k) \in \mathcal{C}$ for all $k \in \mathbb{Z}_{[\bar{h}, \infty)}$. If $r(k)=r$ for all $k \in \mathbb{Z}_{[\bar{h}, \infty)}, r \in \Gamma$, then there exists a finite $\bar{k} \in \mathbb{Z}_{0+}$ such that $v(\bar{k})=r$.

Proof 4 If $(x(\bar{h}), v(\bar{h}-1)) \in \mathcal{O}_{\infty}, v(\bar{h})=v(\bar{h}-1)$ is feasible for (23), by Lemma 14.

Due to (19b) and since for each $k \in \mathbb{Z}_{[\bar{h}, \infty)}, v(k)=v(k-$ 1) is feasible, it follows that $J^{*}(k) \leq\|r-v(k-1)\|_{2}^{2}=$ $J^{*}(k-1)$, where $J^{*}(k)$ denotes the optimal cost at step $k$. Thus, the optimal cost is nonincreasing, and there exists $J^{*} \geq 0$, such that $J^{*}=\lim _{k \rightarrow \infty}\|r-v(k)\|_{2}^{2}$. Note that $v(k)$ is the projection of $r$ on the set defined by (19c). Using the properties of the norm projection on a closed convex set, $\|v(k)-v(k-1)\|_{2}^{2} \leq\|r-v(k-1)\|_{2}^{2}-\| r-$ $v(k) \|_{2}^{2}$, and thus $\lim _{k \rightarrow \infty}\|v(k)-v(k-1)\|_{2}=0$.

Let $x(k+1)=A x(k)+B v(k)+\bar{W} \Delta v(k)$ and $x_{e}(k)=$ $x_{e}(v(k))=A x_{e}(k)+B v(k)$, then $x(k+1)-x_{e}(k)=$ $A\left(x(k)-x_{e}(k)\right)+\bar{W}(v(k)-v(k-1))$. Since A is Schur and $\lim _{k \rightarrow \infty}\|v(k)-v(k-1)\|=0$, it follows that $\lim _{k \rightarrow \infty}\left\|x(k)-x_{e}(k)\right\|=0$.

To complete the proof we note that there exists $\sigma>0$ such that for all $v \in \Gamma,\left(x_{e}(v) \oplus \mathcal{B}(\sigma), v\right) \in \operatorname{int}\left(\mathcal{O}_{\infty}\right)$. Thus, $(x(k), v) \in \mathcal{O}_{\infty}$ and $x(k) \rightarrow x_{e}(k)$ as $k \rightarrow \infty$ imply that $(x(k), v) \in \operatorname{int}\left(\mathcal{O}_{\infty}\right)$ for all $k$ sufficiently large. By the invariance of $\mathcal{O}_{\infty}$, it follows that $(A x(k)+B v, v) \in$ $\operatorname{int}\left(\mathcal{O}_{\infty}\right)$ and there exists $\rho>0$ such that $(A x(k)+$ $B v, v) \oplus \mathcal{B}(\rho) \in \operatorname{int}\left(\mathcal{O}_{\infty}\right)$, for all $k$ sufficiently large.

Consider $v_{\mathrm{fs}}(k)=v(k)+\frac{r-v(k)}{\|r-v(k)\|_{2}} \gamma$. Clearly, for all $k$ sufficiently large, $v_{\mathrm{fs}}(k)$ is feasible for (23) for some $\gamma>$ $\epsilon_{v}>0$, where $\epsilon_{v}$ is finite, yet possibly small. Also, if $\|r-v(k)\| \leq \epsilon_{v}, v(k+1)=r$ is feasible. Thus, for $k$ sufficiently large $\left\|r-v_{\mathrm{fs}}(k)\right\| \leq \max \left\{\|r-v(k)\|-\epsilon_{v}, 0\right\}$. Hence, for $k$ sufficiently large, $0 \leq\|r-v(k+1)\|_{2} \leq$ $\left\|r-v_{\mathrm{fs}}(k)\right\|_{2} \leq \max \left\{\|r-v(k)\|-\epsilon_{v}, 0\right\}$. Thus, there exists a finite $\bar{k} \in \mathbb{Z}_{0+}$ such that $\|r-v(\bar{k}-1)\| \leq \epsilon_{v}$, and hence, $v(\bar{k})=r$.

\section{Network Reference Governor with Output Feedback}

In this section, we relax Assumption 3 into the following.

Assumption $17 C_{m}$ in (7) is such that $\operatorname{rank}\left(C_{m}\right)<n$, and the pair $\left(C_{m}, A\right)$ is observable. 
Under Assumption 17, only output feedback is available, so the state needs to be estimated, and the estimation error has to be accounted for in enforcing the constraints. For enforcing constraints through output feedback in systems subject to general additive disturbances, set-membership observers have been proposed in [34,35]. Since by (11) the uncertainty is proportional to the command variation, here we use a standard observer and compute a bound on the estimation error based on the sequence of past commands. Consider the observer,

$$
\hat{x}(k+1)=A \hat{x}(k)+B v(k)+L\left(y_{m}(k)-C_{m} \hat{x}(k)\right),
$$

where $\hat{x}(k) \in \mathbb{R}^{n}$ is the state vector estimate, and $L$ is the observer gain. Let $A_{L}=A-L C_{m}$ and $\varepsilon(k)=$ $x(k)-\hat{x}(k)$ be the observer error. Under Assumption 12, the observer error dynamics are governed by,

$$
\varepsilon(k+1)=A_{L} \varepsilon(k)+W(\delta) \Delta v(k),
$$

where $\Delta v$ appears in the estimation error dynamics because the delay introduces uncertainty on when the input is applied. In order to account for the estimation error in (6) we enforce a constraint on $\varepsilon(k)$.

Assumption 18 There exists a compact set $\mathcal{E} \subset \mathbb{R}^{n}$, with $0 \in \operatorname{int}(\mathcal{E})$ satisfying,

$$
\begin{aligned}
& C \mathcal{E} \subset \mathcal{C} \\
& A_{L} \mathcal{E} \subset \operatorname{int}(\mathcal{E})
\end{aligned}
$$

By $(26)$, if $\varepsilon(k) \in \mathcal{E}$, then $y(k) \in \mathcal{C}$ at least for $\hat{x}(k)=0$. Also, $\mathcal{E}$ is PI for $(25)$ if $\Delta v=0$. In fact, due to $(26 \mathrm{~b})$ and compactness of $\mathcal{E}$, there exists $\lambda \in \mathbb{R}_{[0,1)}$ such that

$$
A_{L} \mathcal{E} \subseteq \lambda \mathcal{E}
$$

i.e., $\mathcal{E}$ is $\lambda$-contractive for $(25)$ when $\Delta v=0$.

Lemma 19 Let $c^{-} \in \mathbb{R}_{[0,1)}, \varepsilon \in c^{-\mathcal{E}},\left(\hat{x}, v^{-}\right) \in(1-$ $\left.c^{-}\right) \mathcal{O}_{\infty}$. Let $c \in \mathbb{R}_{[0,1)}, v \in \mathbb{R}^{m}$ be such that

$$
\begin{aligned}
& \mathcal{D}(\Delta v) \subseteq\left(c-\lambda c^{-}\right) \mathcal{E} \\
& (A \hat{x}+B v+d, v) \in(1-c) \mathcal{O}_{\infty}, \forall d \in \mathcal{D}(\Delta v)
\end{aligned}
$$

If $\varepsilon(k)=\varepsilon, \hat{x}(k)=\hat{x}, v(k-1)=v^{-}$, and for all $h \in$ $\mathbb{Z}_{[k+1, \infty)}, v(h)=v$, then $y(h) \in \mathcal{C}$, for all $h \in \mathbb{Z}_{[k, \infty)}$.

Proof 5 Let $\mathcal{O}_{\infty}$ be the maximum output admissible set for (5), (6). Then [33, 36], $(1-c) \mathcal{O}_{\infty}$ is the maximum output admissible set for $(5)$ subject to $y(k) \in(1-c) \mathcal{C}$. If $\mathcal{E}$ satisfies $(26 \mathrm{~b}), c \mathcal{E}$ also satisfies $(26 \mathrm{~b})$.

If $\hat{x}(k) \in(1-c) \mathcal{O}_{\infty}$ and $\varepsilon(k) \in c \mathcal{E}$ for some $c \in \mathbb{R}_{[0,1)}$, then $C \hat{x}(k) \in(1-c) \mathcal{C}$ and $C \varepsilon(k) \in c C \mathcal{E} \subseteq c \mathcal{C}$. Thus, $y(k)=C(\hat{x}(k)+\varepsilon(k)) \in(1-c) \mathcal{C} \oplus c \mathcal{C}=\mathcal{C}$.
Since $\varepsilon(k) \in c^{-} \mathcal{E}$, by $(25),(28 \mathrm{a}), \varepsilon(k+1) \in c^{-} A_{L} \mathcal{E} \oplus$ $\mathcal{D}(\Delta v(k)) \subseteq \lambda c^{-} \mathcal{E} \oplus\left(c-\lambda c^{-}\right) \mathcal{E}=c \mathcal{E}$. Since for all $h \in \mathbb{Z}_{[k, \infty)}, v(h)=v$, then $\varepsilon(h+1) \in c A_{L} \mathcal{E} \subseteq \lambda c \mathcal{E} \subseteq$ $c \mathcal{E}$, for all $h \in \mathbb{Z}_{[k, \infty)}$. Thus, since for all $h \in \mathbb{Z}_{[k, \infty)}$, $(\hat{x}(h), v) \in(1-c) \mathcal{O}_{\infty}$, we have $y(h)=C(\hat{x}(h)+\varepsilon(h)) \in$ $(1-c) \mathcal{C} \oplus c C \mathcal{E} \subseteq(1-c) \mathcal{C} \oplus c \mathcal{C}=\mathcal{C}$ for all $h \in \mathbb{Z}_{[k, \infty)}$.

The output feedback netRG law is then defined by

$$
\begin{aligned}
&\left(g_{\varepsilon}\left(x, v^{-}, c^{-}\right), \tilde{c}\right)= \\
& \arg \min _{v \in \Gamma, \tilde{c} \in \mathbb{R}_{[0,1)}}\|r-v\|^{2} \\
& \text { s.t. }(A x+B v+d, v) \in(1-\tilde{c}) \mathcal{O}_{\infty} \\
& \forall d \in \mathcal{D}(\Delta v) \\
& \mathcal{D}(\Delta v) \subseteq\left(\tilde{c}-\lambda c^{-}\right) \mathcal{E}
\end{aligned}
$$

$$
\begin{aligned}
g_{\varepsilon}^{c}\left(v, v^{-}, c^{-}\right)=\arg & \min _{c \in \mathbb{R}_{[0,1)}}\|c\|^{2} \\
& \text { s.t. } \quad \mathcal{D}(\Delta v) \subseteq\left(c-\lambda c^{-}\right) \mathcal{E} .
\end{aligned}
$$

Theorem 20 Consider (5) under Assumption 2, let the command $v(k)$ be subject to random delay satisfying Assumption 12, and let $\mathcal{E}$ satisfy Assumption 18. If at some $\bar{h} \in \mathbb{Z}_{0+}, \varepsilon(\bar{h}) \in \mathcal{E}$ and $(x(\bar{h}), v(\bar{h}-1)) \in \mathcal{O}_{\infty}$, then for the closed loop of (5) and $c(k)=g_{\varepsilon}^{c}(v(k), v(k-1), c(k-$ 1)) with $v(k)=g_{\varepsilon}(x(k), v(k-1), c(k-1))$, it follows that $y(k) \in \mathcal{C}$ for all $k \in \mathbb{Z}_{[\bar{h}, \infty)}$. If $r(k)=r$ for all $k \in \mathbb{Z}_{[\bar{h}, \infty)}$, $r \in \Gamma$, there exists a finite $\bar{k} \in \mathbb{Z}_{0+}$ such that $v(\bar{k})=r . \square$

Proof 6 First, $v(\bar{h})=v(\bar{h}-1), c(\bar{h}+1)=\lambda c(\bar{h})$ is a feasible solution of $(29)$, (30), since $\mathcal{O}_{\infty}$ is $P I, \mathcal{D}(0)=\{0\}$, and by Lemma 19. The reasoning can be repeated for any $k \in \mathbb{Z}_{[\bar{h}, \infty)}$. Thus, by using the same procedure as in the proof of Theorem 16, $J^{*}(k)=\|r-v(k)\|_{2}^{2} \leq J^{*}(k-1)$, and there exists $J^{*} \geq 0, J^{*}=\lim _{k \rightarrow \infty}\|r-v(k)\|_{2}^{2}$. This implies that $\lim _{k \rightarrow \infty}\|v(k)-v(k-1)\|=0, \lim _{k \rightarrow \infty}\left\|x(k)-x_{e}(k)\right\|=$ 0. Furthermore, if $\lim _{k \rightarrow \infty}\|v(k)-v(k-1)\|=0$, $\lim _{k \rightarrow \infty}\|\varepsilon(k)\|=0$, and by $(30), \lim _{k \rightarrow \infty} c(k)=0$.

The rest of the proof can be completed as the proof of Theorem 16, by defining $\mathcal{E}_{k}=c(k) \mathcal{E}$ and noticing that for any $\mathcal{E}_{k}$ compact and $\sigma>0$, by (27) there exists a finite $h \in \mathbb{Z}_{+}$, such that $\mathcal{E}_{k+h} \in \mathcal{B}(\sigma)$.

\section{Network Reference Governor for Long Delay}

Next we develop the netRG for when Assumption 12 does not hold, so that the delay model (1) is considered.

When the network delay becomes large or possibly unbounded, command overtaking is possible. Thus, for a command to be robust it needs to guarantee constraint 
satisfaction for every possible sequence of past commands, which may lead to conservativeness or high computational complexity [29]. In this paper, we avoid excessive conservativeness and complexity by two key elements: $(i)$ the netRG generates a robust command assuming that the delay is within a certain interval; $(i i)$ the receiver verifies the validity of such an assumption on the received command, and discards the invalid commands. Referring to the architecture in Figure 1, such a verification logic is implemented at the plant.

We select $\alpha \in \mathbb{R}_{(0,1)}$ and define

$$
\bar{\delta}=\alpha T_{s}
$$

Assumption 21 Let $\delta_{k} \in \mathbb{R}_{0+}, k \in \mathbb{Z}_{0+}, \delta_{k}$ are i.i.d. ${ }^{1}$ with probability density function, $\phi(\delta): \mathbb{R}_{0+} \rightarrow \mathbb{R}_{0+}$ such that $\int_{0}^{\alpha T_{s}} \phi(\delta) d \delta=q$, for $q \in \mathbb{R}_{(0,1)}$.

Lemma 22 Under Assumption 21 for any finite $\ell \in \mathbb{Z}_{+}$, $\lim _{k \rightarrow \infty} \mathbf{P}\left[\exists h \in \mathbb{Z}_{[0, k]}: \delta_{h+i} \leq \alpha T_{s}, \forall i \in \mathbb{Z}_{[0, \ell-1]}\right]=1$.

Proof 7 Consider an arbitrary choice for $\ell \in \mathbb{Z}_{+}$. The probability of observing $\ell$ consecutive delays of duration less than $\alpha T_{s}$ from the sequence $\left\{\delta_{k}\right\}_{k}$, can be modeled as a Markov chain with states $\left\{s_{j}\right\}_{j=1}^{\ell}$. Each state represents how many delays shorter than $\alpha T_{s}$ occurred since the last delay longer than $\alpha T_{s}$. The transition probabilities are $\mathbf{P}\left[s_{j} \mid s_{j-1}\right]=q, j \in \mathbb{Z}_{[1, \ell]}, \mathbf{P}\left[s_{0} \mid s_{j-1}\right]=1-q$, $j \in \mathbb{Z}_{[1, \ell]}$. Finally, $\mathbf{P}\left[s_{\ell} \mid s_{\ell}\right]=1$ and $\mathbf{P}\left[s_{j} \mid s_{\ell}\right]=0$, for all $j \neq \ell$. Such Markov chain is absorbing [37], with absorbing state $s_{\ell}$, which is reachable from every $s_{j}, j \in \mathbb{Z}_{[1, \ell]}$. Thus, for any finite $\ell \in \mathbb{Z}_{+}, \lim _{k \rightarrow \infty} \mathbf{P}\left[s(k)=s_{\ell}\right]=1$.

Thus, by Lemma 22 for any $\alpha \in \mathbb{R}_{[0,1)}$ a finite sequence of arbitrary length of delays shorter than $\alpha T_{s}$ will eventually occur with probability 1 . The properties of absorbing Markov chains [37] ensure that the average number of steps to observe such a sequence, i.e., $\mathbf{E}\left[k \in \mathbb{Z}_{(\ell, \infty)}\right.$ : $\left.\delta_{k-\ell}>\bar{\delta}, \quad \delta_{k-i} \leq \bar{\delta}, i \in \mathbb{Z}_{[0, \ell-1]}\right]$, and the related variance are finite, and can be computed for any finite $\ell \in \mathbb{Z}_{+}$.

The control and actuation strategy for the netRG that handles longer-than-sampling delays is reported in Algorithm 1 . At any $k \in \mathbb{Z}_{0+}$, the plant sends to the netRG the state $x\left(t_{k}\right)$ and the command being applied $v^{-}\left(t_{k}\right)$. For the current reference $r(k)$, the netRG uses (19) to compute $v(k)$, where $\bar{W}$ is obtained from (18) using $\bar{\delta}$ in (31). Then, $v(k)$ and $r_{v(k)}=r(k)$ are sent to the plant.

Since, $v(k)$ guarantees constraint satisfaction only if $\delta_{k} \leq \alpha T_{s}$, it needs to be verified at the plant to ensure

1 Non i.i.d. delays can be handled similarly, when a property as the one in Lemma 22 can be established. that no constraint violation actually occurs. Such verification is based on $\mathcal{O}_{\infty}$, which is stored at the plant, together with the actuated command at the current time $t, v(t)=v_{a c t}$, and a buffer $\mathbf{v}_{\mathrm{bfr}}$ with all the commands that have been applied during $\left[t_{k}, t\right), t \leq t_{k+1}$.

For a command $v$ received at time $t \in \mathbb{R}_{\left[t_{k}, t_{k+1}\right)}$ together with the corresponding reference $r_{v}$, the verification logic computes $\overline{\Delta v}=\max _{i}\left\|v-\left[\mathbf{v}_{\mathrm{bfr}}\right]_{i}\right\|_{\infty}$, where $\left[\mathbf{v}_{\mathrm{bfr}}\right]_{i}$ is the $i^{\text {th }}$ command currently stored in the buffer. The command $v$ is actuated if and only if,

$$
\begin{aligned}
& \left\|r_{v}-v\right\|_{2}^{2}<\left\|r_{v}-v_{a c t}\right\|_{2}^{2}, \\
& \left((A x(k)+B v) \oplus \mathcal{D}_{\mathrm{T}_{\mathrm{s}}}(\overline{\Delta v}), v\right) \subseteq \mathcal{O}_{\infty}
\end{aligned}
$$

where $\mathcal{D}_{\mathrm{T}_{\mathrm{s}}}(\overline{\Delta v})=\left\{d \in \mathbb{R}^{n}: \exists \delta \in \mathbb{R}_{\left[0, T_{s}\right]}, \quad d=\right.$ $W(\delta) \overline{\Delta v}\}$. While (32a) enforces the reduction of the reference tracking error, $(32 \mathrm{~b})$ enforces that constraints will not be violated. Indeed, the proposed approach requires (32) to be evaluated at the plant. However, (32) only evaluates the inequalities for a given command, while (23) has to select a command that satisfies the inequalities and minimizes the cost function. Hence, the computations performed at the plant are still significantly less than those in the remote controller.

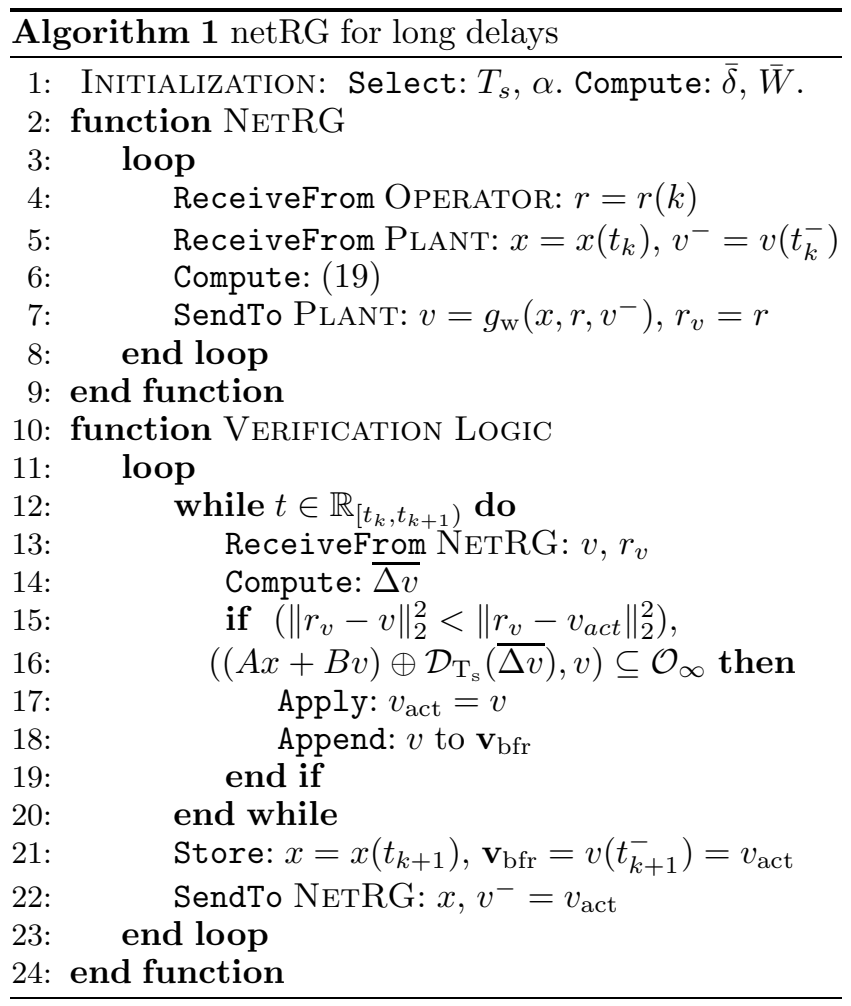

Remark 23 The reference $r_{v}$ is sent together with the command $v$ so that the commands that do not improve tracking, for instance because of packet overtaking, are discarded. This cannot correct for the case where the received command is coupled with an older reference, i.e., 
$r_{v}$ has changed in the meantime. In this case it is still possible to see a tracking performance degradation, because the command may move towards an older value of the reference. However, constraint satisfaction is still guaranteed, and assuming that the references are piecewiseconstant with long periods, which is common in reference governor applications, the performance will self-correct rapidly since all newly generated commands will be received together with the newer value of $r_{v}$.

Theorem 24 Consider (5) under Assumption 2, let the command be subject to a random delay satisfying Assumption 21. If at some $\bar{t} \in \mathbb{R}_{\left[t_{\bar{h}-1}, t_{\bar{h}}\right)}$, v( $\left.\bar{t}\right)$ is applied to the plant such that $\left(x\left(t_{\bar{h}}\right), v(\bar{t})\right) \in \mathcal{O}_{\infty}$, then the closed system obtained by (3) controlled by Algorithm 1 is such

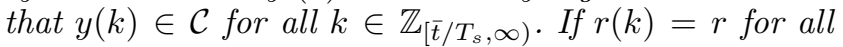
$k \in \mathbb{Z}_{[\bar{h}, \infty)}, r \in \Gamma, \lim _{k \rightarrow \infty} \mathbf{P}\left[\|v(k)-r\|_{2}^{2}=0\right]=1$.

Proof 8 If $\left(x\left(t_{\bar{h}}\right), v(\bar{t})\right) \in \mathcal{O}_{\infty}, y(\tilde{k}) \in \mathcal{C}$ for $\tilde{k} \in \mathbb{Z}_{0+}$, $\tilde{k}=\min _{k>\bar{t} / T_{s}} k$. Furthermore due to $(19)$, if $v(t)=v(\bar{t})$ for all $t \geq \bar{t}, y(k) \in \mathcal{C}$ for all $k \in \mathbb{Z}_{[\tilde{k}+1, \infty)}$. Any future command that is actuated satisfies (32), and it has the same property. Hence, $y(k) \in \mathcal{C}$, for any $k$ such that $k T_{s}>\bar{t}$.

By Theorem 16, if $\delta_{k} \in \mathbb{R}_{[0, \bar{\delta}]}$, for all $k \in \mathbb{Z}_{0+}$, for any $\left(x_{0}, v_{0}^{-}\right) \in \mathcal{O}_{\infty}, r \in \Gamma$ there exists a finite $\bar{k}\left(x_{0}, v_{0}^{-}, r\right) \in \mathbb{Z}_{+}$such that $v\left(\bar{k}\left(x_{0}, v_{0}^{-}, r_{0}\right)\right)=r$.Under Assumption 2, $\mathcal{O}_{\infty}$ is bounded. Hence, there exists $\overline{\bar{k}}=1+\max _{r \in \Gamma,(x, v) \in \mathcal{O}_{\infty}} \bar{k}(x, v, r)$ such that if $\delta_{k} \in \mathbb{R}_{[0, \bar{\delta}]}$ for all $k \in \mathbb{R}_{[0, \overline{\bar{k}}]}, v(\overline{\bar{k}})=r$ for all $\left(x(0), v^{-}(0)\right) \in \mathcal{O}_{\infty}$, $v^{-}(0), r \in \Gamma$. Hence, given $k_{0} \in \mathbb{Z}_{+}$, such that for $x\left(k_{0}\right)$ there exists $v$, for which $\left(x\left(k_{0}\right), v\right) \in \mathcal{O}_{\infty}$, and $\left\{\delta_{k_{0}+i}\right\}_{i=0}^{h}, h \geq \overline{\bar{k}}+1$, such that $\delta_{k_{0}+i} \leq \bar{\delta}, i \in \mathbb{Z}_{[0, h]}$, then $v\left(k_{0}+h\right)=r$. Due to $(32)$, if $v\left(k_{0}+\overline{\bar{k}}\right)=r$, for all $k \geq k_{0}+\overline{\bar{k}}, v(k)=v\left(k_{0}+\overline{\bar{k}}\right)$. Furthermore, due to (11), if $\Delta v(k)=0, x(k+1)$ is independent of $\delta_{k}$, and hence, $\delta_{k_{0}+i}, i \geq \overline{\bar{k}}$, does not affect the dynamics.

Due to Lemma 22 for any $h \in \mathbb{Z}_{[0, \infty)}, \lim _{k \rightarrow \infty} \mathbf{P}\left[\exists k_{0} \in\right.$ $\left.\mathbb{Z}_{[0, k]}: \delta_{k_{0}+i} \leq \alpha T_{s}, \forall i \in \mathbb{Z}_{[0, \bar{h}]}\right]=1$. Thus, $\lim _{k \rightarrow \infty} \mathbf{P}\left[\|v(k)-r\|_{2}^{2}=0\right]=1$.

The properties of absorbing Markov chains [37] also guarantee that the average number of steps $\bar{k}$ such that $v(\bar{k})=r$ is finite, and so is the variance.

\section{Application to Orientation Control of a Spacecraft with a Flexible Appendage}

Often, spacecraft are equipped with an appendage made of lightweight materials, such as a solar panel, a robotic arm, an instrument, an antenna, or a telescope. When the spacecraft is re-oriented, the appendage may bend

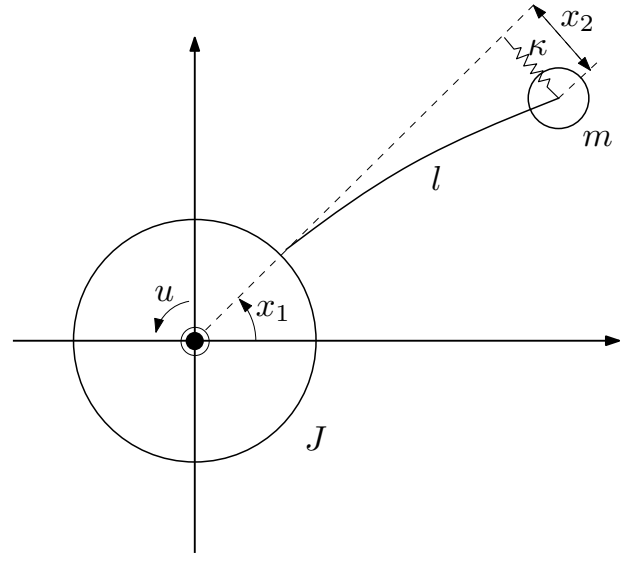

Fig. 3. Model of the spacecraft with flexible appendage.

and oscillate, which can degrade sensitive equipment on the appendage or even cause structural damage if not properly controlled. We consider an agile spacecraft with a flexible appendage that has to be re-oriented with a rotational maneuver about a specified axis.

As shown in Figure 3, the spacecraft with flexible appendage can be modeled as two bodies, the spacecraft bus and the appendage, connected by a flexible shaft. When the shaft bends, the appendage is subject to an elastic force, modeled as if obtained by a spring acting on the displacement from the straight shaft position. The dynamics are described by,

$$
\begin{aligned}
\left(J+m l^{2}\right) \ddot{x}_{1}+m l \ddot{x}_{2} & =u \\
m \ddot{x}_{2}+\kappa x_{2}+m l \ddot{x}_{1} & =0
\end{aligned}
$$

where $x_{1}[\mathrm{rad}]$ denotes the orientation angle of the spacecraft bus, $x_{2}[\mathrm{~m}]$ denotes the linear deflection of the flexible appendage at its tip, and the model parameters are $m=1 \mathrm{~kg}, J=50 \mathrm{kgm}^{2}, \kappa=0.1[\mathrm{~N} / \mathrm{m}], l=40[\mathrm{~m}]$. In order to avoid structural damage, the deflection of the flexible appendage must satisfy,

$$
-0.2 \leq x_{2} \leq 0.2,
$$

and the control torque is limited by,

$$
-0.8 \leq u \leq 0.8
$$

For a command $v$, the plant co-located controller produces the torque $u[\mathrm{Nm}]$ given by the unconstrained linear quadratic regulator,

$$
u=-F_{\mathrm{LQ}} x+G_{\mathrm{LQ}} v
$$

designed to have unitary steady state gain from $v$ to $x_{1}$. Figure 4 shows that for a desired spacecraft angle $r$, without a governor, i.e., $v=r$, the constraints are significantly violated. 

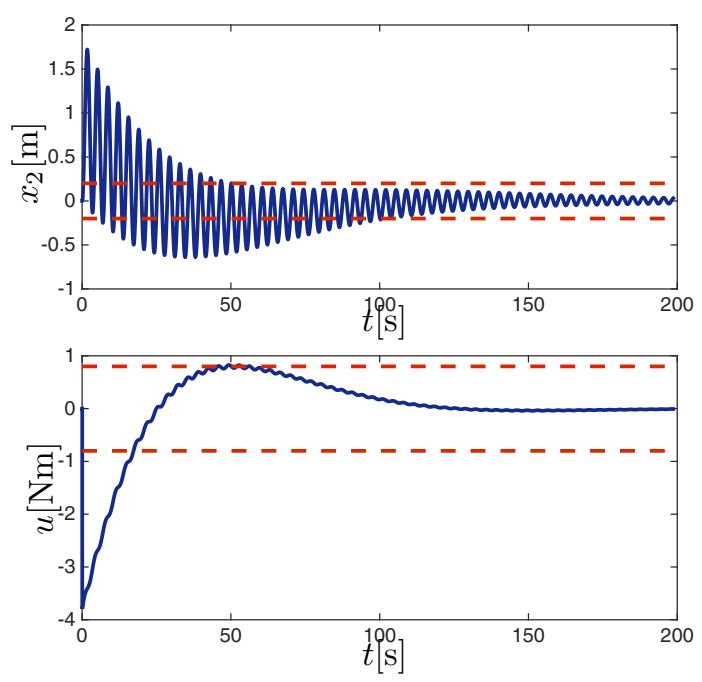

Fig. 4. Change in orientation without reference governor. Top: appendage deflection, $x_{2}$. Bottom: torque, $u$. Constraints: red dash lines.

Considering the cases when the re-orientation maneuver is executed by remotely controlling the spacecraft, we design a netRG that receives $r$ and generates $v$ for $(36)$ to enforce constraints (34), (35). We obtain a discrete-time model (5a) of (33) in closed loop with (36) for $T_{s}=1 \mathrm{~s}$. The constrained outputs (5b) and the constraints (6) are obtained from (33), (34), and (35) for a sampling period of $T_{c}=T_{s} / 4=0.25 \mathrm{~s}$. Constraints upsampling helps limiting intersampling violations, see Remark 9. Although the constraints are enforced every $0.25 \mathrm{~s}$, the update period of the reference governor is still 1.0s.

We first consider the case where the delay in the command channel is shorter than the sampling period, i.e., $\delta(t) \in[0,0.995] \mathrm{s}$ for all $t \geq 0$, and it is random, i.i.d, and uniformly distributed. The netRG is designed as described in Section 3. The results are shown in Figures $5-7$. Figure 5 shows that unlike the conventional reference governor, whose variables are denoted by the superscript $(r g)$, the netRG enforces the constraints despite the time-varying delay. The orientation change maneuver of the netRG is only slightly slowed down with respect to the conventional reference governor, for avoiding constraint violations due to the delay.

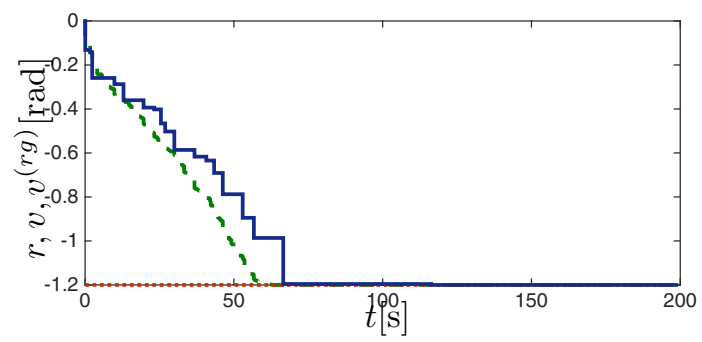

Fig. 5. Change in orientation with short communication delay. Reference, $r$ (dot), and virtual reference, $v$, for conventional reference governor (dash) and netRG (solid).
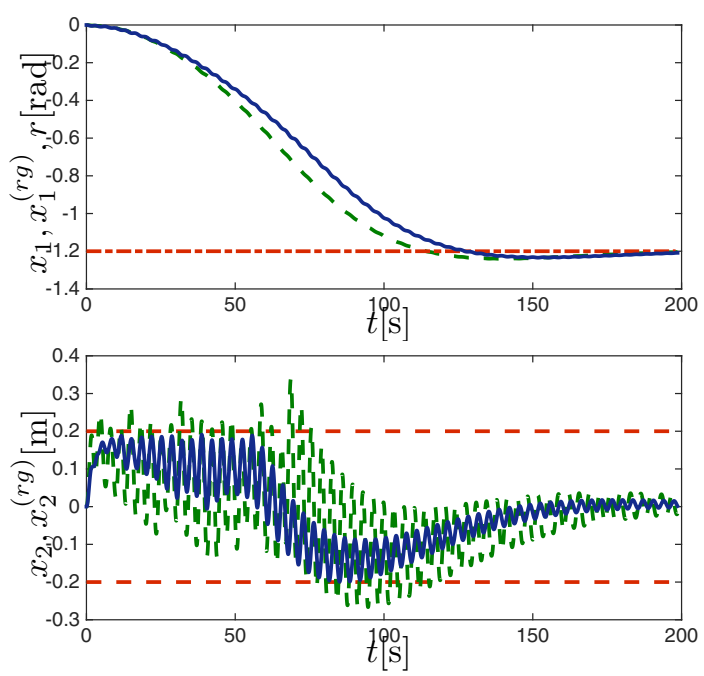

Fig. 6. Change in orientation with conventional reference governor (dash) and netRG (solid) with of short communication delay. Top: spacecraft bus orientation, $x_{1}$, reference, $r$ (dash dot). Bottom: appendage deflection, $x_{2}$. Constraints: red dash lines.

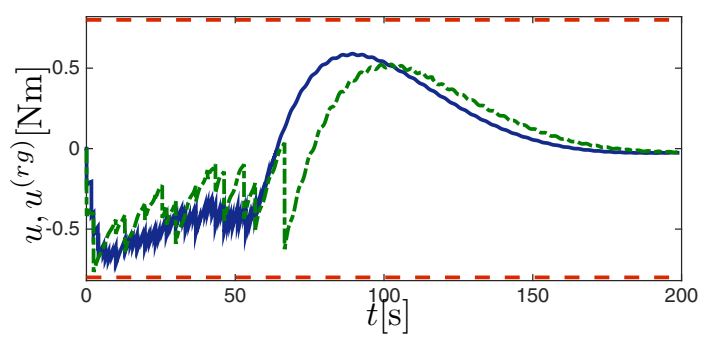

Fig. 7. Change in orientation with conventional reference governor (dash) and netRG (solid) with of short communication delay. Torque, $u$. Constraints: red dash lines.

Next, we consider the case where full state information is not available and an observer is used with the netRG, as described in Section 4. The only available measurement is the bus orientation, $x_{1}$, and the observer gain $L$ is chosen so that $\mathcal{E}$ in Assumption 18 is $\lambda$-contractive, with $\lambda=0.51$ in (27). At the initial time, the system is at rest, the actual estimation error is $\varepsilon(0)=0$, and the estimator parameter $c$ is initialized by $c(0)=0.1$, so that $\varepsilon(0) \in c(0) \mathcal{E}$. The simulation results are shown in Figures 8,9 . Constraints are enforced although the action of the reference governor is more conservative to prevent constraint violations due to the estimation error.

Last, we demonstrate the case where the delay is longer than the sampling period, and specifically $\delta(t) \in[0,10] \mathrm{s}$, for all $t \in \mathbb{R}_{0+}$. The netRG is implemented as described in Section 5, with $\alpha=0.995$, and full state measurement is assumed. The results are shown in Figures 10-11, where the constraints are enforced despite a delay up to 10 times the update period of the netRG. The system response is slower and more conservative than the short delay case, due to the effects of the longer delay, however 

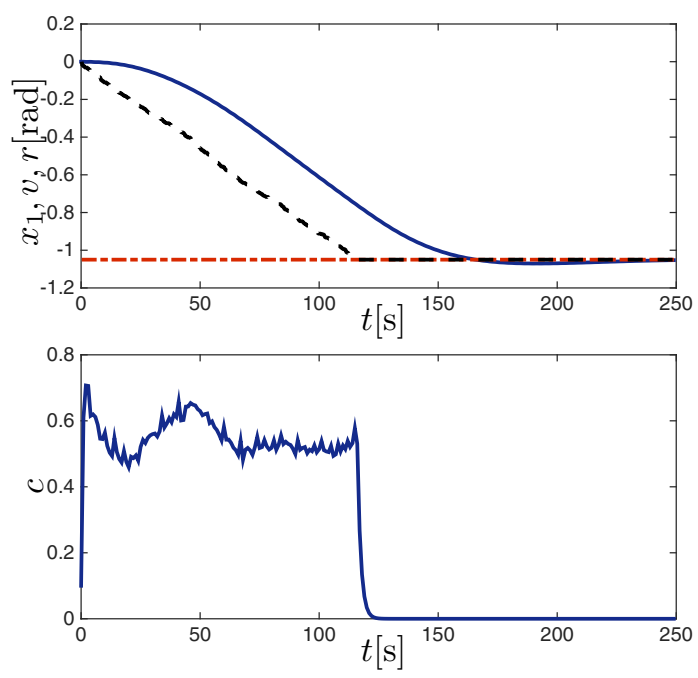

Fig. 8. Change in orientation with output feedback netRG with short communication delay. Top: Reference, $r$ (dot), virtual reference, $v$ (dash), and bus orientation, $x_{1}$ (solid). Bottom: estimation error bounding parameter, $c$.
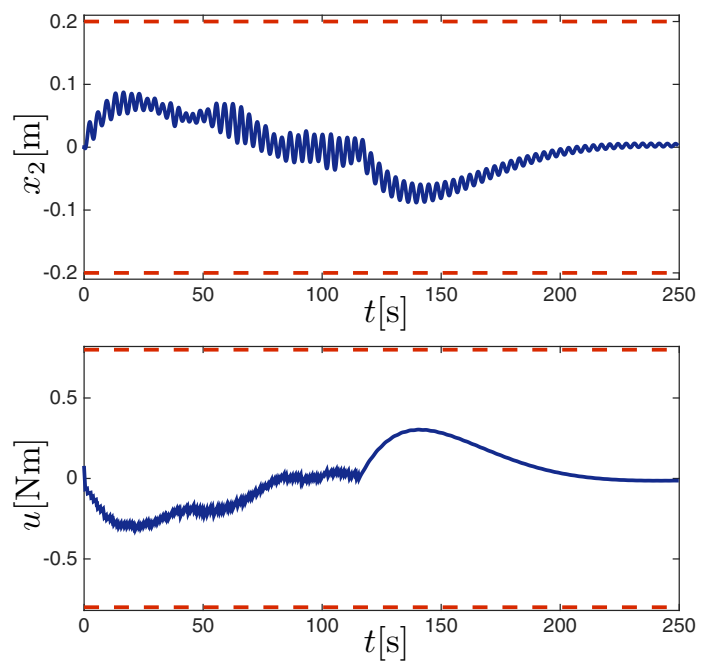

Fig. 9. Change in orientation with output feedback netRG with short communication delay. Top: appendage deflection $x_{2}$. Bottom: torque input $u$. Constraints: red dash lines.

the command $v$ still converges to $r$ in finite time.

\section{Conclusions and Future Research}

We developed a reference governor for systems subject to variable time-delay, such as in network control systems with asynchronous communication. By modeling the delay effect as an additive disturbance with size proportional to the input rate of change, we have introduced a network reference governor that robustly enforces constraints and for which finite-time convergence is guaran-

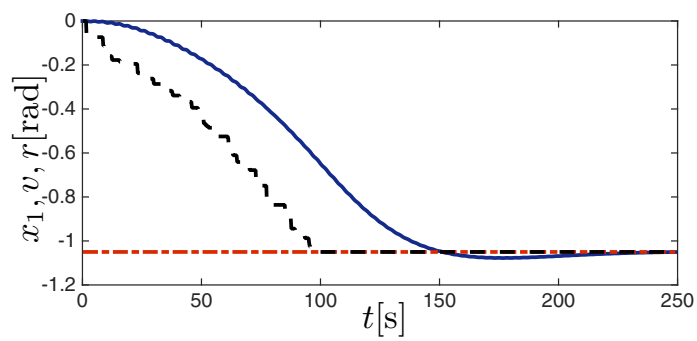

Fig. 10. Change in orientation with netRG with long communication delay. Reference, $r$ (dot), virtual reference, $v$ (dash), and bus orientation, $x_{1}$ (solid).
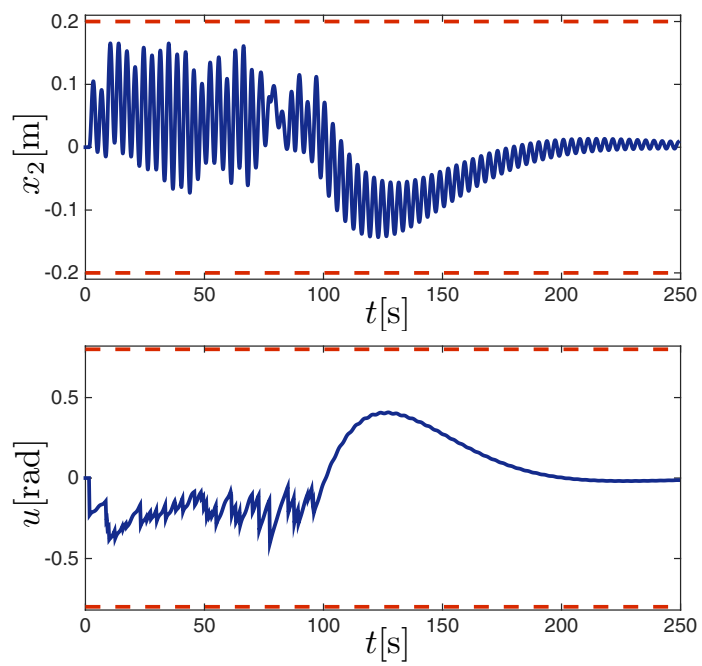

Fig. 11. Change in orientation with netRG with long communication delay. Top: appendage deflection, $x_{2}$. Bottom: torque, $u$. Constraints shown by red dash lines.

teed when the delay is shorter than the sampling period. We extended the method to the cases of output feedback and arbitrarily long, and possibly unbounded, delay. The netRG has been demonstrated on a case study of a remotely controlled re-orientation of a spacecraft with a flexible appendage for different types of delays induced by the communication network. Future research will focus on including delay in the measurement channel following the ideas in [1, III.A], which exploit some degree of equivalence between delays in the command and measurement channels.

\section{References}

[1] S. Di Cairano and I. Kolmanovsky, "Rate limited reference governor for networked controlled systems," in Proc. American Contr. Conf., Baltimore, MD, 2010, pp. 3704-3709.

[2] S. Di Cairano and I. V. Kolmanovsky, "Further developments and applications of network reference governor for constrained systems," in Proc. American Contr. Conf., Montreal, Canda, 2012, pp. 3907-3912.

[3] P. Arcara and C. Melchiorri, "Control schemes for teleoperation with time delay: A comparative study," Robotics and Autonomous Systems, vol. 38, no. 1, pp. 49-64, 2002. 
[4] A. Okamura, "Methods for haptic feedback in teleoperated robot-assisted surgery," Industrial Robot, vol. 31, no. 6, pp. 499-508, 2004.

[5] T. Fong and C. Thorpe, "Vehicle teleoperation interfaces," Autonomous robots, vol. 11, no. 1, pp. 9-18, 2001.

[6] T. Samad, P. McLaughlin, and J. Lu, "System architecture for process automation: Review and trends," Journal of Process Control, vol. 17, pp. 191-201, 2007.

[7] S. Di Cairano, H. Park, and I. Kolmanovsky, "Model predictive control approach for guidance of spacecraft rendezvous and proximity maneuvering," International Journal of Robust and Nonlinear Control, vol. 22, no. 12, pp. 1398-1427, 2012.

[8] C. F. Caruntu, M. Lazar, R. H. Gielen, P. van den Bosch, and S. Di Cairano, "Lyapunov based predictive control of vehicle drivetrains over can," Control Engineering Practice, vol. 21, no. 12, pp. 1884-1898, 2013.

[9] W. Zhang, M. S. Branicky, and S. M. Phillips, "Stability of networked control systems," IEEE Control Systems Magazine, vol. 21, no. 1, pp. 84-99, 2001.

[10] P. Antsaklis and J. Baillieul, "Special issue on networked control systems," IEEE Trans. Aut. Control, vol. 49, no. 9, pp. 1421-1423, Sep. 2004.

[11] K.-E. Årzén, A. Bicchi, G. Dini, S. Hailes, K. H. Johansson, J. Lygeros, and A. Tzes, "A component-based approach to the design of networked control systems," European Journal of Control, 2007.

[12] J. Hespanha, P. Naghshtabrizi, and Y. Xu, "A survey of recent results in networked control systems," Proc. of IEEE Special Issue on Technology of Networked Control Systems, vol. 95 , no. 1 , pp. 138-162, Jan. 2007.

[13] A. Bemporad, S. Di Cairano, E. Henriksson, and K. Johansson, "Hybrid model predictive control based on wireless sensor feedback: an experimental study," Int. J. Robust Nonlinear Control, vol. 20, no. 2, 2010, special issue "Industrial applications of wireless control".

[14] D. Nesic and A. R. Teel, "Input-output stability properties of networked control systems," Automatic Control, IEEE Transactions on, vol. 49, no. 10, pp. 1650-1667, 2004.

[15] D. Liberzon and D. Nesic, "Input-to-state stabilization of linear systems with quantized state measurements," Automatic Control, IEEE Transactions on, vol. 52, no. 5, pp. 767-781, 2007.

[16] N. van de Wouw, P. Naghshtabrizi, M. Cloosterman, and J. P. Hespanha, "Tracking control for sampled-data systems with uncertain time-varying sampling intervals and delays," International Journal of Robust and Nonlinear Control, vol. 20, no. 4, pp. 387-411, 2010.

[17] M. B. Cloosterman, L. Hetel, N. Van De Wouw, W. Heemels, J. Daafouz, and H. Nijmeijer, "Controller synthesis for networked control systems," Automatica, vol. 46, no. 10, pp. 1584-1594, 2010.

[18] R. H. Gielen, S. Olaru, M. Lazar, W. Heemels, N. van de Wouw, and S.-I. Niculescu, "On polytopic inclusions as a modeling framework for systems with time-varying delays," Automatica, vol. 46, no. 3, pp. 615-619, 2010.

[19] D. E. Quevedo and D. Nesic, "Input-to-state stability of packetized predictive control over unreliable networks affected by packet-dropouts," Automatic Control, IEEE Transactions on, vol. 56, no. 2, pp. 370-375, 2011.

[20] N. Elia and J. N. Eisenbeis, "Limitations of linear control over packet drop networks," Automatic Control, IEEE Transactions on, vol. 56, no. 4, pp. 826-841, 2011.
[21] S. Tatikonda and S. Mitter, "Control under communication constraints," IEEE Trans. Aut. Control, vol. 49, no. 7, pp. 1056-1068, 2004.

[22] W. P. M. H. Heemels, A. R. Teel, N. van de Wouw, and D. Nesic, "Networked control systems with communication constraints: Tradeoffs between transmission intervals, delays and performance," Automatic Control, IEEE Transactions on, vol. 55, no. 8, pp. 1781-1796, 2010.

[23] L. Zhang, H. Gao, and O. Kaynak, "Network-induced constraints in networked control systemsa survey," IEEE Trans. Ind. Informatics, vol. 9, no. 1, pp. 403-416, 2013.

[24] P. L. Tang and C. W. de Silva, "Compensation for transmission delays in an ethernet-based control network using variable-horizon predictive control," IEEE Trans. Contr. Systems Technology, vol. 14, no. 4, pp. 707-718, 2006.

[25] I. Kolmanovsky, E. Garone, and S. Di Cairano, "Reference and command governors: A tutorial on their theory and automotive applications," in Proc. American Contr. Conf., 2014, pp. 226-241.

[26] M. Pachter and R. Miller, "Manual flight control with saturating actuators," IEEE Control Systems Magazine, vol. 18, no. 1, pp. 10-20, 1998.

[27] J. Cook, J. Grizzle, and J. Sun, "Automotive control systems," Control System Applications, p. 87, 1999.

[28] A. Bemporad, "Predictive control of teleoperated constrained systems with unbounded communication delays," in Proc. 37th IEEE Conf. on Decision and Control, Tampa, FL, 1998, pp. 2133-2138.

[29] A. Casavola, M. Papini, and G. Franze, "Supervision of networked dynamical systems under coordination constraints," IEEE Trans. Aut. Control, vol. 51, no. 3, pp. 421-437, March 2006.

[30] E. Gilbert and I. Kolmanovsky, "Nonlinear tracking control in the presence of state and control constraints: a generalized reference governor," Automatica, vol. 38, no. 12, pp. 20632073, 2002

[31] A. Bemporad, A. Casavola, and E. Mosca, "Nonlinear control of constrained linear systems via predictive reference management," IEEE Trans. Aut. Control, vol. 42, no. 3, pp. 340-349, Mar 1997.

[32] E. Garone, F. Tedesco, and A. Casavola, "Sensorless supervision of linear dynamical systems: The feed-forward command governor approach," Automatica, vol. 47, no. 7, pp. 1294-1303, 2011.

[33] E. Gilbert and K. Tan, "Linear systems with state and control constraints: the theory and applications of maximal output admissible sets," IEEE Trans. Aut. Control, vol. 36, no. 9, pp. 1008-1020, 1991.

[34] A. Bemporad and A. Garulli, "Output-feedback predictive control of constrained linear systems via set-membership state estimation," Int. J. Control, vol. 73, no. 8, pp. 655-665, 2000.

[35] D. Angeli, A. Casavola, and E. Mosca, "On feasible set-membership state estimators in constrained command governor control," Automatica, vol. 37, no. 1, pp. 151-156, 2001.

[36] I. Kolmanovsky and E. Gilbert, "Theory and computation of disturbance invariant sets for discrete-time linear systems," Mathematical Problems in Engineering, vol. 4, no. 4, pp. 317-363, 1998.

[37] C. M. Grinstead and J. L. Snell, Introduction to probability. Providence, RI: American Mathematical Soc., 1998. 